\title{
Focal plane metrology for the LSST camera
}

\author{
Andrew P. Rasmussen ${ }^{a}$, Layton $\mathrm{Hale}^{b}$, Peter Kim ${ }^{a}$, Eric Lee ${ }^{a}$, Martin Perl ${ }^{a}$, Rafe Schindler ${ }^{a}$, \\ Peter Takacs ${ }^{c}$, Timothy Thurston ${ }^{a}$ and the LSST Camera Team \\ ${ }^{a}$ Stanford Linear Accelerator Center, 2575 Sand Hill Road, Menlo Park, CA 94025, U.S.A.; \\ ${ }^{b}$ Innovative Machine Solutions, 11501 Dublin Blvd. Suite 200, Dublin, CA 94568, U.S.A.; \\ ${ }^{c}$ Brookhaven National Laboratory, P.O. Box 500, Upton, NY, 11973, U.S.A.
}

\begin{abstract}
Meeting the science goals for the Large Synoptic Survey Telescope (LSST) translates into a demanding set of imaging performance requirements for the optical system over a wide $\left(3.5^{\circ}\right)$ field of view. In turn, meeting those imaging requirements necessitates maintaining precise control of the focal plane surface $(10 \mu \mathrm{m} \mathrm{P}-\mathrm{V})$ over the entire field of view $(640 \mathrm{~mm}$ diameter $)$ at the operating temperature $\left(\mathrm{T} \sim-100^{\circ} \mathrm{C}\right)$ and over the operational elevation angle range. We briefly describe the heirarchical design approach for the LSST Camera focal plane and the baseline design for assembling the flat focal plane at room temperature. Preliminary results of gravity load and thermal distortion calculations are provided, and early metrological verification of candidate materials under cold thermal conditions are presented. A detailed, generalized method for stitching together sparse metrology data originating from differential, non-contact metrological data acquisition spanning multiple (non-continuous) sensor surfaces making up the focal plane, is described and demonstrated. Finally, we describe some in situ alignment verification alternatives, some of which may be integrated into the camera's focal plane.
\end{abstract}

Keywords: Telescopes, LSST, Wide Field Imaging, Sensor Array, Focal Plane, Flatness, Metrology, Finite Element Metrology, Stitching Algorithms

\section{INTRODUCTION}

The Large Synoptic Survey Telescope (LSST) will perform high observing efficiency, seeing-limited imagery, with an exceptionally large étendue $\left(\mathrm{A} \Omega \sim 300 \mathrm{~m}^{2} \mathrm{deg}^{2}\right)$ in several spectral bands. LSST's science requirements ${ }^{1,2}$ are derived from a set of performance parameters needed to meet the divers science objectives outlined for the mission. ${ }^{3}$ Among these, perhaps the most challenging is the imaging performance required for studying Dark Energy via weak lensing of galaxies. This demands an imaging point spread function with very little uncalibrated instrumental signature down to the individual pixel $\left(0.2^{\prime \prime}\right)$ scale over the full $3.5^{\circ}$ field of view (FOV).

LSST's strategy to maintain this imaging quality across the camera's focal plane will be provided by two enabling features: active optical compensation and a well defined focal plane. The active optics requires monitoring the image formation wavefront at multiple field positions distributed over the FOV, ${ }^{4}$ while the focal plane definition and stability will be achieved by adopting precision engineering principles and precision assembly techniques. ${ }^{5}$ The focal plane is required to be flat under well defined operational conditions $\left(\mathrm{T} \sim-100^{\circ} \mathrm{C}\right.$, elevation angle $\left.>45^{\circ}\right)$, but is constructed out of a large number $(\sim 200)$ of individual imaging sensors, assembled in a heirarchical fashion.

In this paper we describe plans and procedures for performing the metrology necessary for ensuring fabrication and operation of the LSST focal plane that will permit realization of the broad science goals of the LSST. We discuss our metrological approaches for the relevant entries in the Science Requirements Document (SRD) and describe how we intend to meet those goals.

Further author information: (Send correspondence and color electronic version requests to A.P.R.) A.P.R.: E-mail: arasmus@slac.stanford.edu, Telephone: 16509262794 


\section{REQUIREMENTS}

An enabling feature of the telescope's large étendue is a fast beam $(\mathrm{F} / 1.25)$ that converges at points across the large field of view (FOV). The focal plane is flat and $\sim 640 \mathrm{~mm}$ in diameter, and is positioned after three corrector lenses and one (selectable) wide-band color filter, all of which are housed in the camera, ${ }^{5}$ while three mirrors of the telescope's main optical system provide the converging beam. Because of the annular geometry of the primary mirror, the depth of field of the system more closely resembles that of a significantly faster beam (F/1.0) in the case of a filled aperture. Consequently, any defocus of the system will appear to degrade the image by a proportional amount (2/3) of the defocus distance. A small degree of non-flatness in the focal plane, therefore, can impart a local defocus to the system and a consequential blur associated with that defocus. Other contributions that can degrade imaging performance, notably distortions to the optics due to changing gravity and thermal load, and probable active optical compensation limitations and are discussed in greater detail in companion papers in these proceedings ${ }^{4,6}$ and are considered to be of comparable magnitude. Throughout this paper, by "non-flat" we mean any deviation of the sensor light entrance surface from the ideal system plane defined by the ideally co-aligned optical elements in the system optical chain.

\subsection{Flatness}

An extensive error budget has been assembled for LSST's baseline imaging performance. Among the various terms, blur due to focal plane non-flatness has a small, but significant, contribution. The corresponding maximum permissible focal plane non-flatness there is $10 \mu \mathrm{m}(\mathrm{P}-\mathrm{V})$ over the entire focal plane area $\left(\sim 3200 \mathrm{~cm}^{2}\right)$. This is in general a daunting task: As compared to the production of the WFI camera mounted to the MPG/ESO $2.2 \mathrm{~m}$ telescope, ${ }^{7}$ the LSST focal plane is approximately 25 times the surface area and requires 4 times tighter tolerances than their measured, as built (warm) focal plane configuration. Any way of arriving within specifications for the LSST focal plane would be acceptable in principle. However, because of the large number of sensors that are ganged together in a heirarchical fashion, ${ }^{5}$ we adopted a breakdown of the non-flatness requirement on the component and subsystem levels. This is summarized in Table 1. To estimate contribution from a distributed mass, focal plane integrating structure or optical bench, we performed finite element analysis computations for a strawman structure design together with different choices of material. These are summarized in Figure 2 and Table 2. An allocation for contribution by the operational environment of the focal plane is based on scaling the largest FEA derived deviations by a factor of a few, and loosens the non-flatness specification from 8 to $10 \mu \mathrm{m}(\mathrm{P}-\mathrm{V})$ between the as built and operational allocations tabulated. In turn, the as built allocation of $8 \mu \mathrm{m}$ $(\mathrm{P}-\mathrm{V})$ is based on precise assembly of $\sim 20$ rafts that individually meet a tighter allocation of $6.5 \mu \mathrm{m}(\mathrm{P}-\mathrm{V})$, each of which are precisely assembled using packaged sensors that individually meet allocations of $5 \mu \mathrm{m}(\mathrm{P}-\mathrm{V})$. Preparation, alignment and metrology of these raft units is described in greater detail in a separate paper in these proceedings. ${ }^{8}$

Table 1. Outline of the budget allocation for focal plane non-flatness.

\begin{tabular}{|c|c|c|c|}
\hline Description & Budget Term & Specifications & Comments \\
\hline $\begin{array}{l}\text { FP max. permissible } \\
\text { non-flatness }\end{array}$ & $10 \mu \mathrm{m}(\mathrm{P}-\mathrm{V})$ & LSST SRD & Top Level Requirement \\
\hline Sensor level & $5 \mu \mathrm{m}(\mathrm{P}-\mathrm{V})$ & operating, cold, $0-45^{\circ}$ tilt & as packaged, ready to mount onto raft \\
\hline Raft level & $6.5 \mu \mathrm{m}(\mathrm{P}-\mathrm{V})$ & operating, cold, $0-45^{\circ}$ tilt & $\begin{array}{l}\text { as packaged, ready to mount into IS } \\
9 \text { sensors assembled onto raft }\end{array}$ \\
\hline FP assembly level & $8 \mu \mathrm{m}(\mathrm{P}-\mathrm{V})$ & cold & $\begin{array}{l}\text { cables and thermal straps attached } \\
21 \text { rafts assembled onto IS }\end{array}$ \\
\hline FP operational level & $10 \mu \mathrm{m}(\mathrm{P}-\mathrm{V})$ & operating, cold, $0-45^{\circ}$ tilt & $\begin{array}{l}\text { Meets top level requirement } \\
\text { gravity load \& thermo-mechanics }\end{array}$ \\
\hline
\end{tabular}




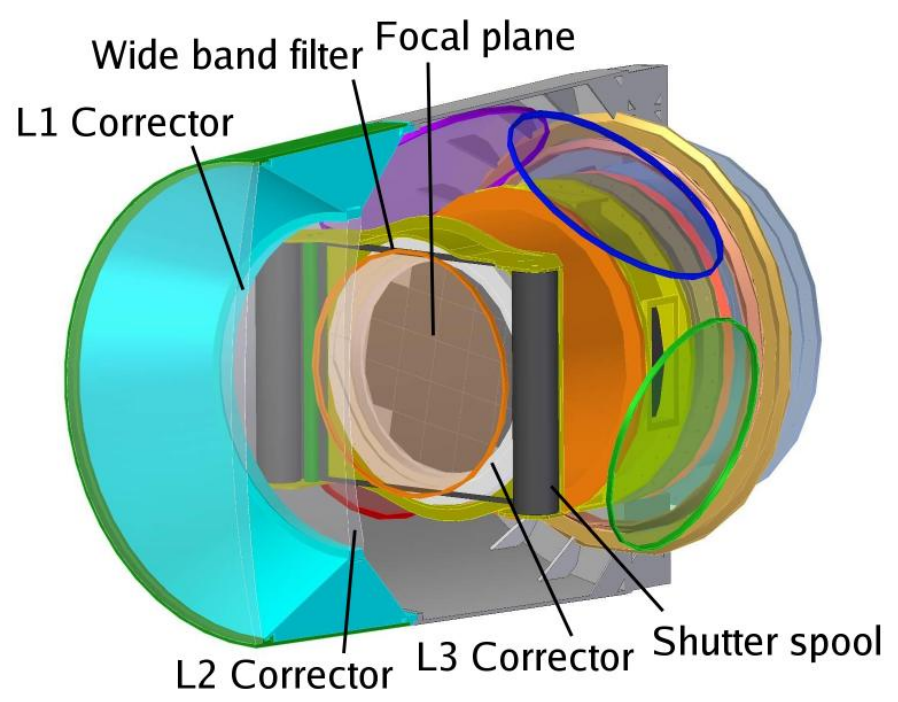

Figure 1. Cut-away schematic of the LSST camera. The lens labeled "L3" is also the vacuum barrier of the cryostat. The focal plane sits directly behind L3.

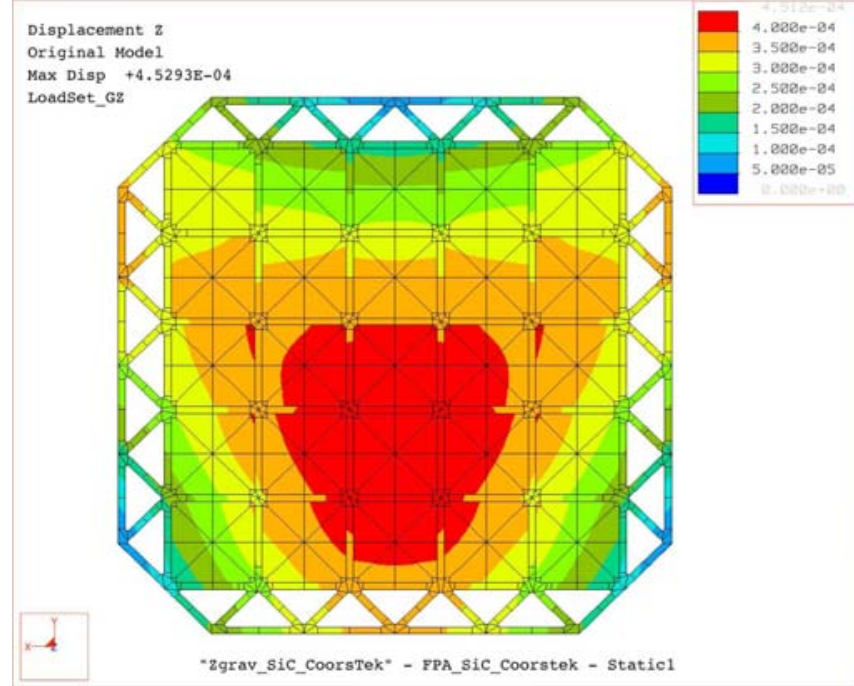

Figure 2. A finite element analysis prediction of out-of-plane height distortion, a difference map between horizontal and vertical orientation of an LSST focal plane strawman. This map shows a $0.25 \mu \mathrm{m} \mathrm{P}-\mathrm{V}$ distortion in the case of SiC material choice tabulated in Table 2. There are 3 assumed mounting points, located at the center top and the two lower corners of the focal plane integrating structure. 
Table 2. Integrating structure (IS) material comparison matrix. Finite element analysis calculations were performed for a strawman design, whose results are tabulated here. A three point mounting constraint was assumed, and deflections due to changing gravitational load were calculated for different material choices. Probable internal stresses and their associated distortions were not calculated. While the silicon carbide ( $\mathrm{SiC}$ ) material choice appears to have superior overall characteristics (high stiffness to density ratio, high thermal conductivity to thermal expansion coefficient ratio), we baselined an operational contribution to focal plane non-flatness that was several times worse than the contribution expected for the metal materials ( $c f$. Table 1).

\begin{tabular}{|c|l|l|l|l|}
\hline Property & Unit & Alum. & Invar 36 & $\mathrm{SiC}$ \\
\hline \hline Total mass with rafts $(25 \mathrm{~kg})$ & $\mathrm{kg}$ & 100 & 246 & 112 \\
\hline \hline P-V gravity sag over aperture & & & & \\
\hline $0-90^{\circ}$ elevation & $\mu \mathrm{m}$ & 1.350 & 1.400 & 0.250 \\
\hline $30-90^{\circ}$ elevation & $\mu \mathrm{m}$ & 0.675 & 0.700 & 0.125 \\
\hline $45-90^{\circ}$ elevation & $\mu \mathrm{m}$ & 0.395 & 0.410 & 0.073 \\
\hline $60-90^{\circ}$ elevation & $\mu \mathrm{m}$ & 0.181 & 0.188 & 0.033 \\
\hline \hline Mode shape and frequency & & & & \\
\hline Mode 1, torsion/twist & $\mathrm{Hz}$ & 205 & 184 & 463 \\
\hline Mode $2, \mathrm{X}$ translation & $\mathrm{Hz}$ & 241 & 217 & 546 \\
\hline Mode 3, Y translation & $\mathrm{Hz}$ & 339 & 321 & 775 \\
\hline Mode 4, Z translation & $\mathrm{Hz}$ & 366 & 346 & 846 \\
\hline \hline Elastic modulus/density & $\mathrm{SI}$ & 25.56 & 17.67 & 130.16 \\
\hline \hline Thermal conductivity/CTE & $\mathrm{SI}$ & 10.00 & 8.08 & 75.00 \\
\hline
\end{tabular}

Flatness assurance of the LSST focal plane will be achieved, therefore, by requiring strict adherence to allocations permitted at the various levels summarized in Table 1, and also by incorporating an in situ means by which to verify focal plane geometry. Specifically, in regards to the assembly aspect, we concentrate on the allocation for assembly of the specification compliant sensor rafts $(6.5 \mu \mathrm{m} \mathrm{P}-\mathrm{V})$ into the specification compliant focal plane assembly $(8 \mu \mathrm{m} \mathrm{P}-\mathrm{V})$. These are the main subjects of this paper. In the case of this focal plane, the metrology and assembly are intimately connected, and describing one of these aspects without the other rapidly loses value.

Metrology of the 2-dimensional pixel registration across all focal plane sensor devices, combined with well understood charge transport (either tight fidelity or precise characterization) is an additional science requirement for LSST's astrometric performance. This aspect of focal plane metrology is beyond the scope of this paper and is not discussed at all here.

\section{ASSEMBLY}

A natural decision point in planning the design for the LSST camera comes in adopting either of two distinct approaches for establishing flatness of the focal plane. These approaches include dynamic actuation compensation of the sub-assemblies and thermo-mechanical build compensation. The first approach would be one where individual components (e.g. packaged sensors) and/or individual sub-assemblies (e.g. assembled rafts) are pistoned and tilted relative to their kinematic mounts to establish co-planarity simultaneously between focal plane constituent surfaces and with the defined system focal plane. Three actuators per sub-assembly (with adequate travel per actuator) would be sufficient to align sub-assemblies to the nominal focal plane surface. This approach would resemble working examples of active optical compensators on segmented primary mirror assemblies, such as those of the Magellan Telescopes ${ }^{9}$ or that planned for the Thirty Meter Telescope. ${ }^{10}$ 
Active compensation for the focal plane raises several issues:

1. Actuators must function in the extreme and clean environment of the camera focal plane, which is cold $\left(-100^{\circ} \mathrm{C}\right)$, under vacuum, and no possibility may be introduced for contamination by foreign material that may measurably alter performance characteristics of the sensors.

2. Depending on level at which actuation is performed, a very large number of actuators will need to be controlled ( $\sim 60$ in the case of actuated rafts, $\sim 600$ in the case of actuated sensors). The number of degrees of freedom for the actuation mechanism quickly becomes unweildy and will require a similar number of precision measurements to perform alignment.

3. As in active optical compensation, an actively compensated focal plane requires feedback. Feedback mechanism may be based either on differential, internal calibrators* referenced to well defined fiducials adjacent to the focal plane, or on local imaging performance. The latter approach may work, but risks circularizing the problem, since active optical compensation is performed relative to a well defined focal plane. The former approach introduces more well characterized instruments to operate in the extreme and clean environmental conditions typical of the focal plane. Feedback could alternatively be provided by an in situ metrology verification system, discussed in $\S 5$.

Common, high precision actuator choices, such as piezoelectrics and pico-motors, possess unique characteristics (also strengths and weaknesses) in terms of their application to the problem. Because of the perceived risks (in complication, functionality as well as in contamination), we do not favour an actuated focal plane, unless it becomes absolutely necessary to meet LSST's science requirements. Therefore, we have taken the approach of fabricating, characterizing and assembling a focal plane that will be specification compliant under operational conditions. This is our baseline approach. As we discuss in the following sections, a variety of possibilities for its production are available but each one relies on a baseline set of assumptions:

1. The heirarchical assembly of the focal plane: the integrating structure carrying tens of raft subassemblies and the raft subassemblies each carrying 9 packaged sensors with their support infrastructure, possesses at every level, certain thermo-mechanical detail that will be measured, traced and compensated for.

2. At the packaged sensor level, this detail includes the intrinsic non-flatness of the sensor at room temperature, any combined thermal bi-layer effect of the device and thermally induced changes of the kinematic mount holding the sensor, as the mounted, packaged sensor's temperature is varied from ambient to operational temperature.

3. At the raft focal plane subassembly level, this detail includes similar combined non-flatnesses, but this time includes intrinsic non-flatness of the sensor mounting points on the raft at room temperature and changes in these points as the raft temperature is varied from ambient to operational temperature.

4. At the focal plane integrating structure (IS) level, this detail includes the non-flatness of the raft subassembly mounting points on the IS and changes to these points as the IS temperature is varied from ambient to operational temperature.

5. We assume that the kinematic mounting approach is stress-free in that it does not induce measurable distortions to the mounted part, both at the packaged sensor and raft subassembly level.

6. We assume that any thermo-mechanical hysteresis is comparable or smaller than the level that is measurable. Alternatively, we assume that thermal cycling will yield repeatable results. Predictability of individual parts is a central requirement for our fabrication approach.

*Internal calibrator possibilities include, for example, capacitive edge sensors coupled with each actuator, or an array of independently defined straight-edges in the array, coupled with sensors that can reference off of those straight-edges. 


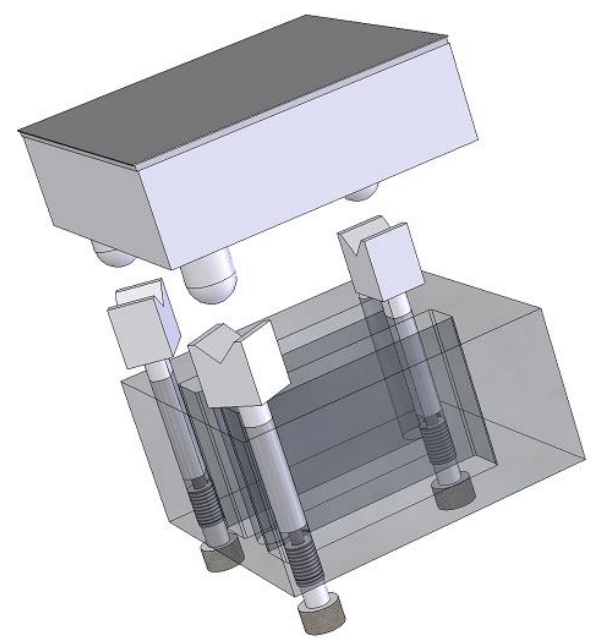

Figure 3. A schematic for a spring loaded kinematic mount that would hold each sensor in a raft subassembly to the raft. A similar concept applies to fixing the raft subassemblies to the focal plane IS.

Due to the various risks posed (e.g., ESD and contamination), handling of assembled focal plane subassembly rafts will be strictly limited to essential operations. However, because of the geographically distributed property of the LSST project, metrology of specification compliant raft focal plane subassemblies will naturally be checked at the time of receipt, but only at ambient temperature, as this will provide adequate verification of metrology performed at the time of assembly.

In the following, we outline a straightforward scheme of measurements and geometric calculations that provides a basis by which we plan to achieve flatness of the LSST focal plane within specification. It includes high precision measurements of at least one standard fixture used in receipt verification metrology of the raft subassemblies, precision measurements of each specification compliant focal plane subassembly raft when mounted to the standard fixture(s), and also precision measurements of the integrating structure under various physical conditions. One important requirement of this approach is for the mounting interface to be identical (as possible) across sub-assemblies, with only piston $(z)$ adjustment available on one side at each contact point. Figure 3 depicts an example of the mating kinematic mount that we envision between mounted sensors and the raft subassembly. ${ }^{8}$ A spring load will assure minimal stress and uniform pressure at each contact point and also an exactly constrained configuration for the mounted object. Similar kinematic mounts are considered for joining each raft subassembly to the integrating structure. The available piston adjustment refered to above is depicted here as a differential screw but may be in the form of a precision post or standoff that is longitudinally compressed by the local spring-induced load.

By taking these essential metrological and (measured) thermo-mechanical details of constituent parts into account, the focal plane will be assembled so that it will meet the flatness specifications when operational. This means that the sensor surfaces may be significantly out of spec at the time of assembly, but specifically within spec under operational conditions. The metrological bookkeeping required to arrive at the desired focal plane figure when assembled and operational listed here, in top-down order.

1. As-built non-planarity of mounting surfaces of the IS.

2. Thermally induced shape variation in the IS. We expect these to be due to variations in internal stress and possible bi-layer effects where brazes or welds were performed.

3. Gravity load induced shape variation in the IS. Finite element calculations were performed to predict the magnitude of these variations, as described in Fig. 2 and Table 2. With proper material choice, these 


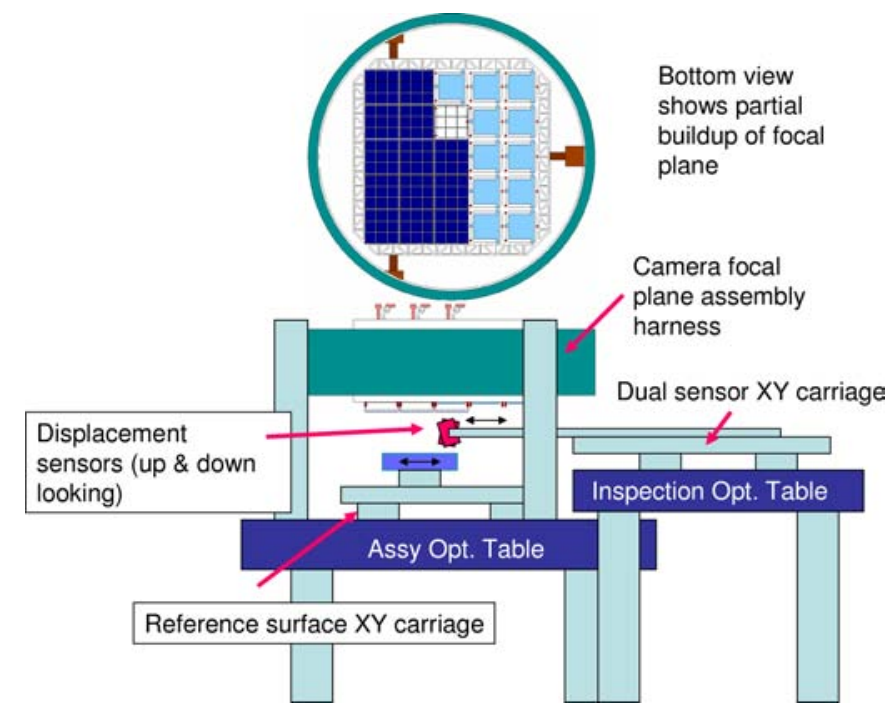

Figure 4. A cartoon representation for performing assembly and alignment verification after attachment of each delivered raft subassembly. In this, two independent non-contact displacement sensors are used to measure the distance between a reference surface (bottom) and a portion of the assembled focal plane (top). Such measurements would be combined according to the general stitching approach described and demonstrated in $\S 4.3$. A more straightforward, perhaps less flexible approach would incorporate a large, polished granite surface and an actuated (X-Y) air bearing carriage for a single displacement sensor ( $c f . \S 4.2)$.

variations can be limited to values well below our error budget allocation.

4. The figure signature of each individual raft, measured at room temperature. As-fabricated sensor nonflatness, combined with thermally induced variations in sensor figure is required to be bounded by the error budget allocation. Typical as-built non-flatness and thermal distortions will be studied using dead silicon, packaged identically to the science sensor packaging.

5. Thermally induced changes to the kinematic mounts that join individual raft subassemblies to the IS.

Table 3 summarizes the list of metrology data required for the constituent subassemblies, how these data will be acquired, and how the data will be used to build the focal plane sensor array. A linear combination of these measurements, and their measured changes due to thermal changes, will be combined to form a room temperature, as built figure map according to which the raft subassemblies will be aligned to and assembled on the focal plane IS.

\section{METROLOGY CONSTRAINTS}

\subsection{Tools}

Because of the risks associated with handling the LSST sensors, we consider only non-contact metrology probes to provide metrological data. Furthermore, because of the number of individual sensor surfaces making up the focal plane, we cannot rely on only normal-incidence, Fizeau interferometry with long coherence lengths: The figure height ambiguity that exists across focal plane discontinuities are too great. Frequency scanning interferometry, white light interferometry and oblique illumination interferometry are possible methods for partially removing figure height ambiguities, but also potentially provide far greater figure accuracy than required. These interferometric methods each require capture of multiple interferograms, so additional sources of systematics (due to room air structure variations) would need to be contained. Furthermore, the sensor level flatness requirement is ambiguous enough so that very high fringe densities may exist (and potential frustration in figure computation), even for parts meeting specifications. 
Table 3. Metrological data required for assemblying the LSST Focal Plane.

\begin{tabular}{|c|c|c|}
\hline Symbol/Measurement & Description & Comments \\
\hline $\begin{array}{l}\zeta_{\mathrm{MF}}\left(x_{h}, y_{h}\right) \\
\rho_{i}\left(x_{k}, y_{k}\right)\end{array}$ & $\begin{array}{l}\text { surface sample for raft metrology } \\
\text { fixture mounting point } h \\
\text { datum sample } k \text { acquired during } \\
\text { metrology of raft } i\end{array}$ & $\begin{array}{l}\text { mapping is performed at ambient } \\
\text { temperature. } \\
\text { " " }\end{array}$ \\
\hline $\begin{array}{l}\zeta_{i}\left(x_{j}, y_{j}\right) \\
\rho_{i}\left(x_{k}, y_{k}\right)\end{array}$ & $\begin{array}{l}\text { sensor array surface sample } j \text { for } \\
\text { raft } i \\
\text { datum sample } k \text { acquired during } \\
\text { metrology of raft } i\end{array}$ & $\begin{array}{l}\text { mapping is performed at ambient } \\
\text { and at operational temperature. } \\
\text { " " }\end{array}$ \\
\hline$\Delta \zeta_{i}\left(x_{j}, y_{j} \mid T_{\text {cold }}, T_{\text {warm }}\right)$ & $\begin{array}{l}\text { thermal distortion function sam- } \\
\text { ple } j \text { for raft } i\end{array}$ & $\begin{array}{l}\text { map of datum-plane subtracted } \\
\text { difference between cold }\left(-100^{\circ} \mathrm{C}\right) \\
\text { and ambient }\left(20^{\circ} \mathrm{C}\right)\end{array}$ \\
\hline $\begin{array}{l}\zeta_{\mathrm{IS}}\left(x_{m}, y_{m}\right) \\
\rho_{\mathrm{IS}}\left(x_{n}, y_{n}\right)\end{array}$ & $\begin{array}{l}\text { surface sample for IS raft mount- } \\
\text { ing point } m \\
\text { IS datum sample } n \text { acquired dur- } \\
\text { ing measurement for raft mount- } \\
\text { ing point } m\end{array}$ & $\begin{array}{l}\text { sampling is performed at ambient } \\
\text { and at operational temperature } \\
\text { " " }\end{array}$ \\
\hline$\Delta \zeta_{\mathrm{IS}}\left(x_{m}, y_{m} \mid T_{\text {cold }}, T_{\text {warm }}\right)$ & $\begin{array}{l}\text { thermal distortion function sam- } \\
\text { ple for raft mounting point } m\end{array}$ & $\begin{array}{l}\text { map of datum-plane subtracted } \\
\text { difference in mounting point be- } \\
\text { tween cold }\left(-100^{\circ} \mathrm{C}\right) \text { and ambient } \\
\left(20^{\circ} \mathrm{C}\right)\end{array}$ \\
\hline
\end{tabular}

We therefore consider using other non-contact dispacement sensors, such as those used by other groups that have built multiple CCD focal planes. ${ }^{7,11}$ A number of sensors types, including laser triangulation displacement sensors, chromatic and non-chromatic confocal displacement sensors, have been considered. Each sensor type generally possess unique strengths and weaknesses, as well as more or less fixed ratios between measurement ranges and linearity measures. For purposes of ongoing R\&D work, we have decided to use three different models of laser triangulation displacement sensors provided by Keyence. These, mounted on ganged translation stages (X-Y; X-Y-Z), will permit non-contact metrology measurements with typical accuracies required.

\subsection{Measurments}

We plan to perform such non-contact metrology under various environments, ranging from pre-assembly parts metrology (room temperature; under vacuum; under vacuum and cold) to assembly time alignment verification to (gravity) load induced sag verification. We have verified, using raytracing, that some of the non-contact sensors selected can be used to verify focal plane flatness changes even while peering through the L3 corrector optic (which also serves as a vacuum barrier), although measurements will have better fidelity if the vacuum barrier thickness is minimized. There are a number of possibilities for choice of the reference surface (with respect to which the focal plane figure height will be measured). Using a monolithic, polished granite block that exceeds both focal plane flatness requirements and focal plane size is one possibility: An X-Y actuated air bearing chuck would be used to carry the non-contact sensor and the entire focal plane surface could be mapped relative to the air bearing carriage surface. Use of the air bearing approach would probably be limited to horizontal orientation of the focal plane.

Another approach is also under consideration, which involves differential measurements between the focal plane and a much smaller reference surface (such as an optical flat), which can be translated independently of the displacement sensor assembly. This configuration is depicted as a cartoon representation in Figure 4. According to this method, sub-samples of the focal plane figure height would be measured rapidly (modulo an arbitrary plane introduced by translation of the reference surface. The rapid measurement of a finite area may 


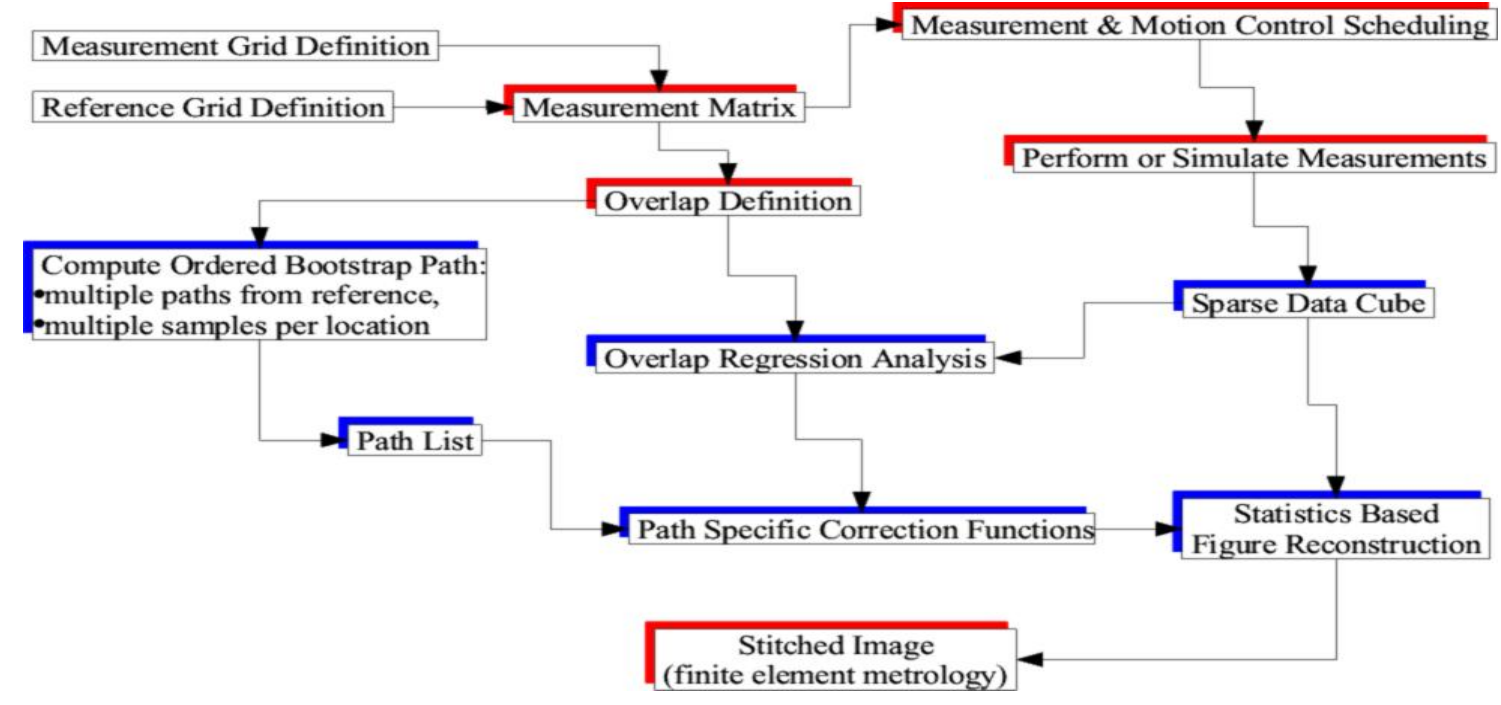

Figure 5. A flowchart representation of the generalized stitching algorithm demonstrated in this contribution. Shadow colors indicate the approximate dimensionality of each operation or intermediate data set. We plan to re-define the algorithm to include two new features. First, the Overlap Definition will be made from contents of the Sparse Data Cube instead of from the Measurement Matrix; Second, Outlier (bootstrapped) measurements will be used to identify problematic reference locations. Figure 7 represent the computed error distributions prior to the Statistics Based Figure Reconstruction step; Figure 8 represents Finally, Figure 9 is a test comparison of the final, Stitched Image produced using finite element metrology, against the figure input.
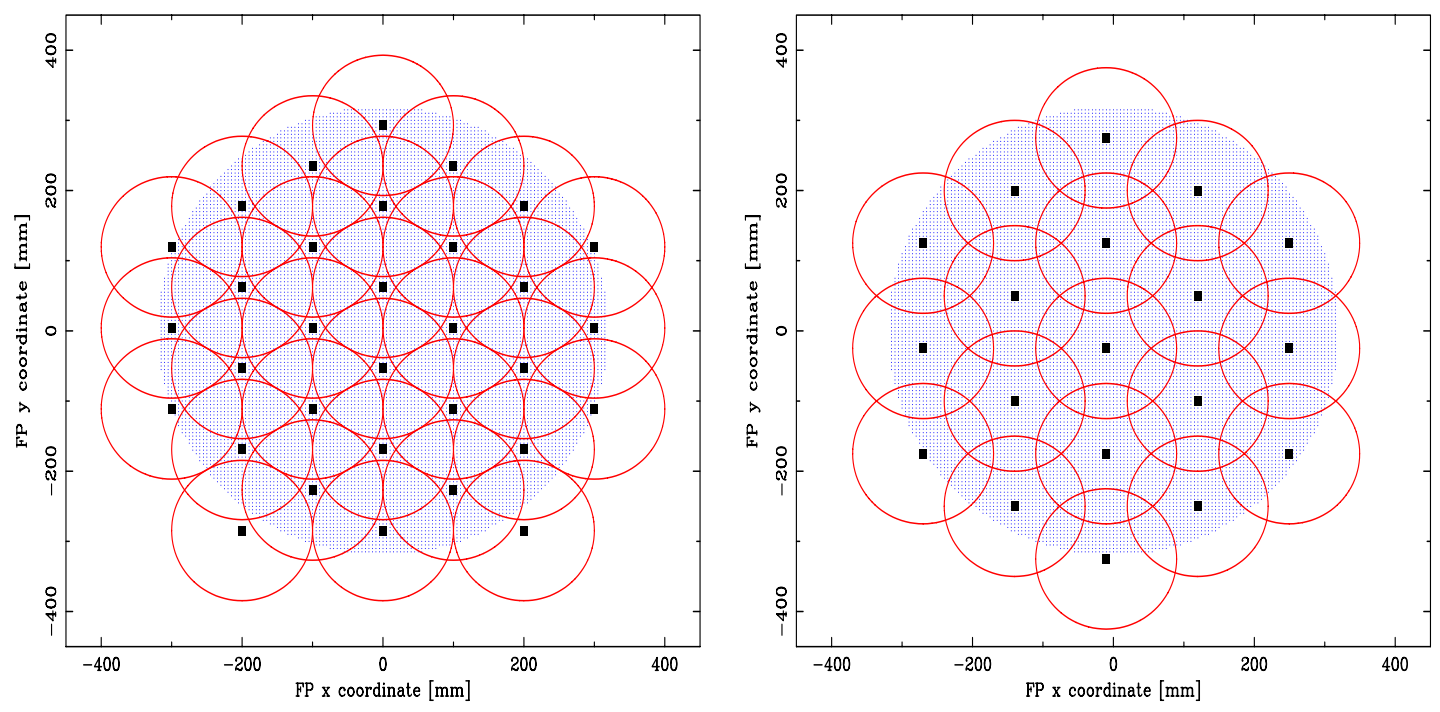

Figure 6. Two possible reference spacing grids considered for the demonstration of the stitching algorithm here. The reference spacing is $2 / \sqrt{3} \times R_{\text {ref }}$, on the left, whereas it is $1.50 \times R_{\text {ref }}$ on the right.

prove to be a useful feature, since accurate measurements can be frustrated by time dependent effects routinely observed wherever sub-micron scale metrology is performed over larger areas. The method naturally requires a high fidelity figure height stitching operation, in which adjacent measurements are combined together to form a full figure height map. Such an operation is described briefly and demonstrated by simulation here. 


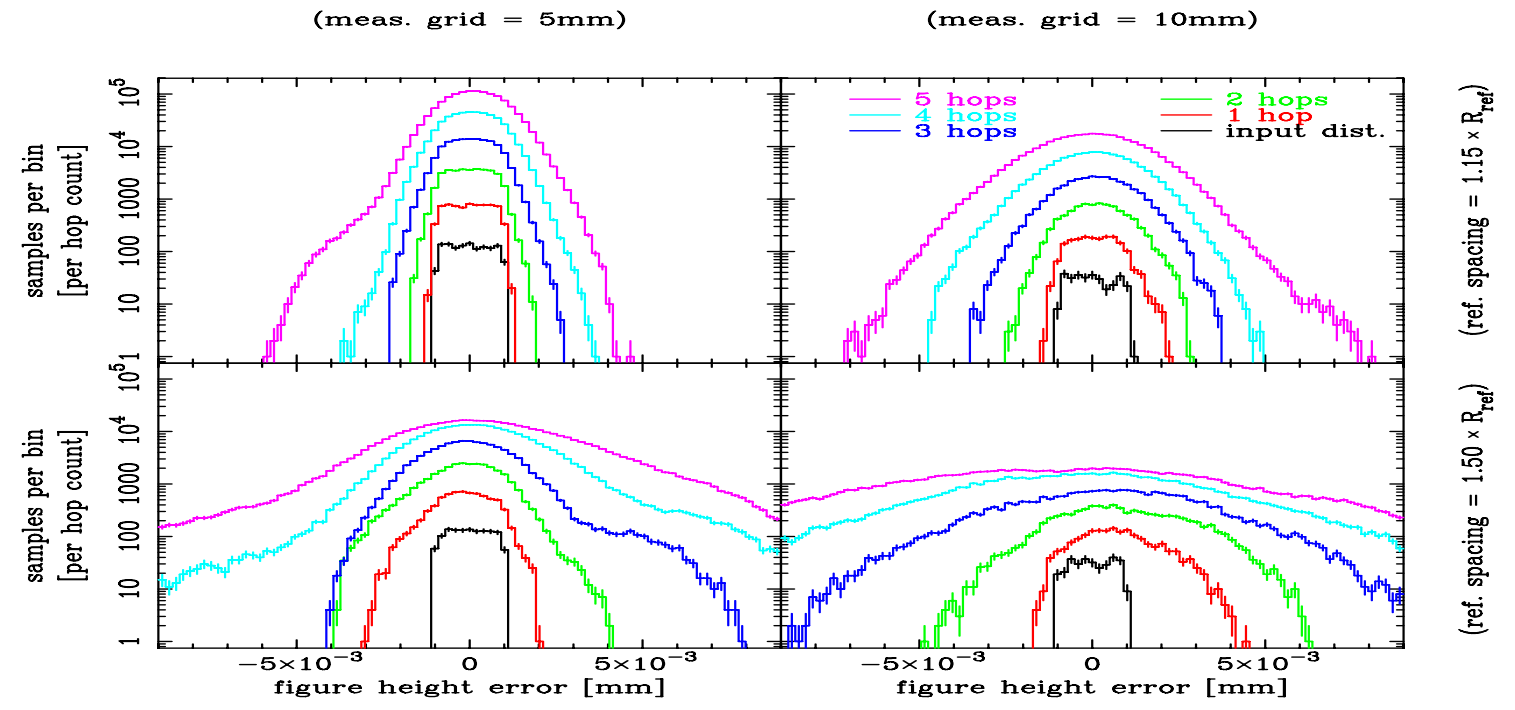

Figure 7. A graphic comparison of the measured error distributions, sorted by the number of hops in the various bootstrap paths. Hop counts range from zero (sampling the input error distribution with no intermediate reference locations; lowest amplitude histograms) to 5 (4 intermediate reference locations and 10 bootstrap planar regression operations; highest amplitude histograms). The four plots represent four separate realizations of measuring a perfectly flat surface using a $100 \mathrm{~mm}$ radius optical flat as a reference in differential mode. Two different measurement grids were used (5 mm grid, left hand plots; $10 \mathrm{~mm}$ grid, right hand plots) and two different reference spacings were used $\left(2 / \sqrt{3} \times R_{\text {Ref }}\right.$ reference spacing, top plots; $1.50 \times R_{\text {Ref }}$ reference spacing, bottom plots). Pictorial representations of the two reference grids are given in Figure 6, and resulting, error distribution width parameters of the bootstrapping averages are displayed in Figure 8. Degradation of the multi-hop bootstrapped height estimates are at least in part compensated for by the larger number of bootstrapping paths available.

\subsection{Large Aperture Figure Height Synthesis}

The general approach envisioned is outlined in the flowchart shown in Figure 5. This describes how the measurement is planned and executed, and how on-the-fly decisions to perform planar regression on sub-samples of the reference aperture are made. The bootstrapping algorithm provides hundreds of estimators for each measurement point on the focal plane. Figure 6 provides representations for two separate reference spacing grids, in units of the reference radius. These reference spacing grids are referred to in the following figures. Figure 7 is a comparison of the sampling distribution generated for four separate realizations of measuring a known figure height function. The degradation induced by the stitching algorithm depends on the number of "hops", or intermediate reference positions, but also on the measurement and reference spacing grids. The input, assumed measurement distribution is also shown there for comparison. The large number of sample estimators (based on different bootstrap paths) provide several ways to compute a single figure height for each location on the measurement grid. In this demonstration, only mean values of all available data (using uniform weighting) were calculated, although median calculations would also provide robust estimators. The distribution of these mean values are represented in Figure 8, where 50 and $90 \%$ width measures are plotted as a function of focal plane radius, for different measurement and reference spacing grid realizations. We note that only moderate degradation of the input error distribution occurs when (1) the reference spacing grid is close to the spacing described by $d_{\mathrm{rg}} \sim 2 / \sqrt{3} \times R_{\text {ref }}$ (ensuring $\sim 30 \%$ overlap between adjacent measurements), and (2) the measurement grid remains small (ensuring at least 1200 samples per reference location). Finally, Figure 9 provides an example of comparing a stitched, synthetic measurement of a test input figure (a cubic term along a single axis) and retrieval of the shape coefficient. 

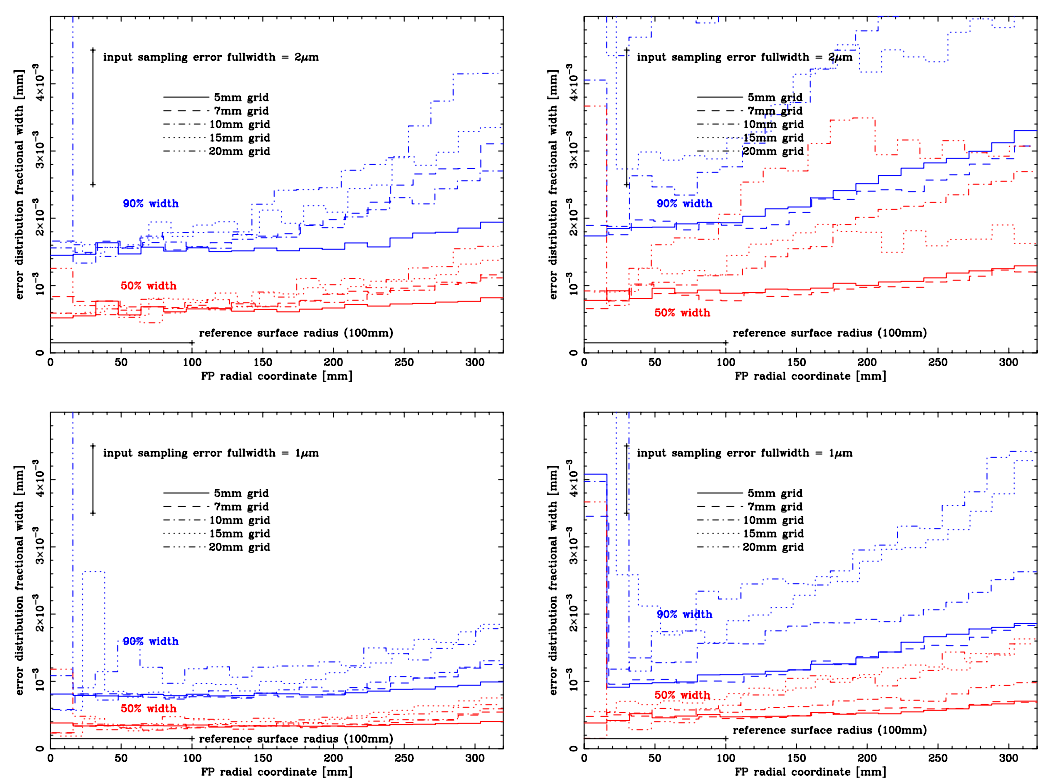

Figure 8. Resulting error distribution width measures derived from various realizations of the measurement matrix, reference spacing grid, input sampling error full-width, et cetera. The width parameters are based on the distribution of errors, this time each location in the measurement grid is represented by a single number (the mean of all possible bootstrap paths with uniform weighting). Reduction of either the measurement grid sampling density or (more importantly) the reference spacing grid density, results in a loss of measurement fidelity. Maintaining the densities of each sampling grid above some critical value appears to ensure high fidelity stitching of the figure to values comparable to the input measurement error distribution.

\section{IN SITU METROLOGY}

Finally, several methods for providing in situ metrology verification are under consideration for possibly incorporation into the focal plane assembly. These would provide independent estimates for changes in the focal plane figure height function. Such internal alignment feedback systems would be crucial in the case of an actively compensated focal plane, but less so if there are no internal actuators for adjustments. Three chief approaches are being considered, and they are only briefly mentioned here.

\subsection{Laser Straightedge}

Relative movement of the sensor raft subassemblies can be measured by incorporating a laser straightedge system, in which ten separate beams are set up to pass beneath the focal plane sensors. Each of 21 rafts then has access to two fiducial beams, nearly orthogonal to one another. Each of the two beams is sensed at two locations in the raft, providing enough data to constrain changes in the plane of the raft with respect to the fiducial beams. Low reflectivity, high transmission pellicles would be used as pick-off mirrors to divert a fraction of the incident beam onto small, video format sensors, permitting the beams to pass on to the next raft. Four variable unknown parameters for each beam (20) and three unknown parameters per raft (63) combine to form 83 unknowns, would be almost exactly constrained by the 4 measurements data points per raft (84). Additional beam sensors can be installed to over-constrain the fit, or permit partial failure of some of the beam sensors.

Sub-micron centroiding capability of such beams using video format cameras, together with camera operation and pellicle deformation at low temperature, and implications of more electronics located inside of the focal plane cryostat are being studied.

\subsection{Capacitive Edge Sensing}

Differential motion between adjacent rafts can be measured accurately and repeatably using capacitive edge sensors. Four sensors (and readout electronics) would be installed per raft edge facing a neighboring raft (128 edge 


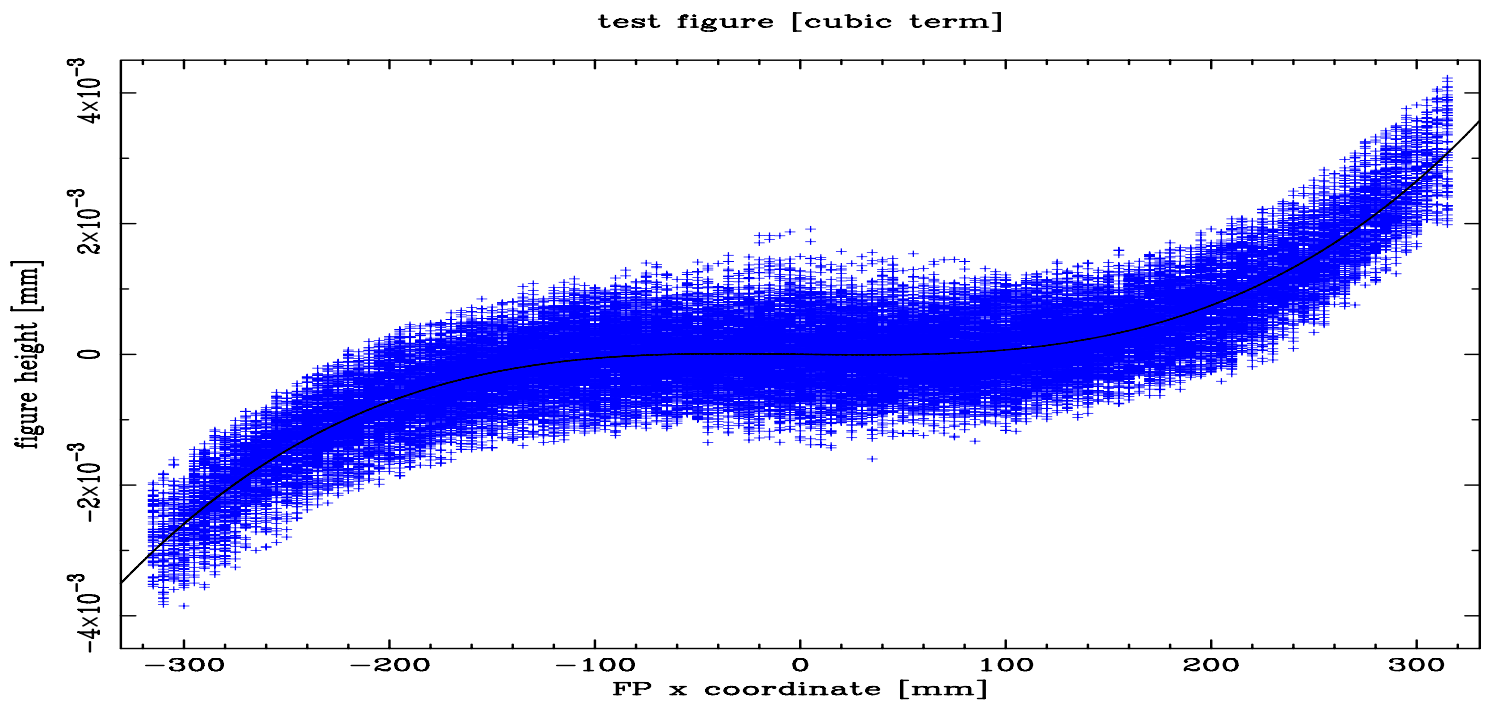

Figure 9. Test of the stitching algorithm. A measurement grid with $5 \mathrm{~mm}$ spacing (12850 locations over $640 \mathrm{~mm}$ diameter) was sampled using 32 different reference locations. All measurements were simulated assuming a 100 mm reference radius, and a measurement error distribution full width of $2 \mu \mathrm{m}$ was assumed. The reference grid spacing used was $2 / \sqrt{3}$ times the reference radius. Because of the $\sim 30 \%$ sampling overlap between reference positions, figure heights at each measurement grid coordinate were computed (on average) over 130 different ways. The mean values for each set of figure heights are plotted here as individual points. The input figure for this trial calculation was $z(x)=\alpha \times x^{3}$, with randomly generated piston $(0-200 \mu \mathrm{m})$ and tilt $\left(0-6^{\prime \prime} ; 0-10^{\prime \prime}\right)$ values for each reference position. The input value for $\alpha$ used was $1 \times 10^{-10} \mathrm{~mm}^{-2}$. The figure values were referenced off of the regression plane for the reference measurement closest to $(x, y)=(0,0)$. The polynomial model shown is a fit to the resulting figure height estimate data, which recovered the cubic coefficient $(\alpha)$ to within $1.1 \%$ of its input value $\left(1.011 \times 10^{-10} \mathrm{~mm}^{-2}\right.$ vs. $\left.1 \times 10^{-10} \mathrm{~mm}^{-2}\right)$.

sensor pairs per focal plane) thereby providing differential piston and differential tilt measures. The accuracy of the capacitive edge sensor system vastly exceeds our focal plane flatness control requirement. Thermally induced variations in response of the electronics is under study.

\subsection{Beam Diffraction Pattern}

Changes in the focal plane figure height function can potentially be measured directly on the sensor surfaces by illuminating a well defined, 2-dimensional diffraction pattern on to the sensor array from several different positions with acute angles of incidence. This is a potentially elegant solution to the problem because it requires no more instruments in the cryostat, but only lasers and gratings. Differentiating vertical displacements from lateral ones naturally require at least two diffraction beams from each grating, illuminating each raft. Sensitivity to vertical displacements is naturally high, due to the acute incidence angle from each diffracted beam. Control of diffracted spot size and propagation of measurement errors into figure height change measurements are currently under investigation.

\section{ACKNOWLEDGMENTS}

The LSST design and development activity is supported by the National Science Foundation under Scientific Program Order No. 9 (AST-0551161) through Cooperative Agreement AST-0132798. Portions of this work were performed in part under Department of Energy contracts DE-AC02-76SF00515, DE-AC02-98CH10886, DE-FG02-91ER40677 and W-7405-Eng-48. Additional funding comes from private donations, in-kind support at Department of Energy laboratories and other LSSTC Institutional Members. 


\section{REFERENCES}

1. S. M. Kahn and LSST Camera Team, "Science Requirements for the Design of the LSST Camera," American Astronomical Society Meeting Abstracts 207, pp. -+, Dec. 2005.

2. D. K. Gilmore, S. M. Kahn, M. Nordby, D. L. Burke, P. O'Connor, J. Olivier, V. Radeka, T. Schalk, and R. Schindler, "LSST camera system overview," in Ground-based and Airborne Instrumentation for Astronomy, Iye and McLean, eds., Proc. SPIE 6269, 2006.

3. J. A. Tyson, Z. Ivezic, S. Kahn, M. Strauss, C. Stubbs, D. Sweeney, and LSST Collaboration, "Science Opportunities with LSST," American Astronomical Society Meeting Abstracts 207, pp. -+, Dec. 2005.

4. C. F. Claver, W. J. Gressler, V. L. Krabbendam, S. S. Olivier, D. L. Phillion, L. G. Seppala, and R. S. Upton, "LSST wavefront sensing and alignment system," in Opto-Mechanical Technologies for Astronomy, Antebi, Atad-Ettedgui, and Lemke, eds., Proc. SPIE 6273, 2006.

5. M. Nordby, L. Hale, and LSST Camera Team, "The Mechanical Design of the LSST Camera," American Astronomical Society Meeting Abstracts 207, pp. -+, Dec. 2005.

6. S. S. Olivier, L. G. Seppala, D. K. Gilmore, L. C. Hale, and W. T. Whistler, "LSST camera optics," in Opto-Mechanical Technologies for Astronomy, Antebi, Atad-Ettedgui, and Lemke, eds., Proc. SPIE 6273, 2006.

7. D. Baade, K. Meisenheimer, O. Iwert, J. Alonso, T. Augusteijn, J. Beletic, H. Bellemann, W. Benesch, A. Boehm, H. Boehnhardt, J. Brewer, S. Deiries, B. Delabre, R. Donaldson, C. Dupuy, P. Franke, R. Gerdes, A. Gilliotte, B. Grimm, N. Haddad, G. Hess, G. Ihle, R. Klein, R. Lenzen, J.-L. Lizon, D. Mancini, N. Muench, A. Pizarro, P. Prado, G. Rahmer, J. Reyes, F. Richardson, E. Robledo, F. Sanchez, A. Silber, P. Sinclaire, R. Wackermann, and S. Zaggia, "The Wide Field Imager at the 2.2-m MPG/ESO telescope: first views with a 67-million-facette eye.," The Messenger 95, pp. 15-16, 1999.

8. P. Z. Takacs, P. O'Connor, V. Radeka, G. Mahler, and J. C. Geary, "LSST detector module and raft assembly metrology concepts," in Opto-Mechanical Technologies for Astronomy, Antebi, Atad-Ettedgui, and Lemke, eds., Proc. SPIE 6273, 2006.

9. H. M. Martin, R. G. Allen, B. Cuerden, S. T. DeRigne, L. R. Dettmann, D. A. Ketelsen, S. M. Miller, G. Parodi, and S. Warner, "Primary mirror system for the first Magellan telescope," in Proc. SPIE Vol. 4003, p. 2-13, Optical Design, Materials, Fabrication, and Maintenance, P. Dierickx, ed., pp. 2-13, July 2000.

10. A. Segurson and G. Z. Angeli, "Computationally efficient performance simulations for a thirty-meter telescope (TMT) point design," in Optimizing Scientific Return for Astronomy through Information Technologies, S. C. Craig and M. J. Cullum, eds., Proc. SPIE 5497, pp. 329-337, Sept. 2004.

11. Y. Komiyama, S. Miyazaki, M. Yagi, N. Yasuda, S. Okamura, M. Sekiguchi, M. Doi, K. Shimasaku, F. Nakata, H. Furusawa, M. Kimura, M. Ouchi, M. Hamabe, and H. Nakaya, "Suprime-Cam: Subaru prime focus camera," in Instrument Design and Performance for Optical/Infrared Ground-based Telescopes, M. Iye and A. F. M. Moorwood, eds., Proc. SPIE 4841, pp. 152-159, Mar. 2003. 